ARTICLE

Received 28 Dec 2015 | Accepted 13 Sep 2016 | Published 20 Oct 2016

DOI: $10.1038 /$ ncomms13206

OPEN

\title{
A direct-sensing galactose chemoreceptor recently evolved in invasive strains of Campylobacter jejuni
}

Christopher J. Day ${ }^{1}$, Rebecca M. King ${ }^{1, \star}$, Lucy K. Shewell ${ }^{1, \star}$, Greg Tram ${ }^{1}$, Tahria Najnin ${ }^{1}$, Lauren E. Hartley-Tassell ${ }^{1}$, Jennifer C. Wilson ${ }^{2}$, Aaron D. Fleetwood ${ }^{3}$, Igor B. Zhulin ${ }^{3,4} \&$ Victoria Korolik ${ }^{1,2}$

A rare chemotaxis receptor, Tlp11, has been previously identified in invasive strains of Campylobacter jejuni, the most prevalent cause of bacterial gastroenteritis worldwide. Here we use glycan and small-molecule arrays, as well as surface plasmon resonance, to show that TIp11 specifically interacts with galactose. Tlp11 is required for the chemotactic response of C. jejuni to galactose, as shown using wild type, allelic inactivation and addition mutants. The inactivated mutant displays reduced virulence in vivo, in a model of chicken colonization. The TIp11 sensory domain represents the first known sugar-binding dCache_1 domain, which is the most abundant family of extracellular sensors in bacteria. The Tlp11 signalling domain interacts with the chemotaxis scaffolding proteins $\mathrm{CheV}$ and $\mathrm{CheW}$, and comparative genomic analysis indicates a likely recent evolutionary origin for TIp11. We propose to rename TIp11 as CcrG, Campylobacter ChemoReceptor for Galactose.

\footnotetext{
${ }^{1}$ Institute for Glycomics, Griffith University, Gold Coast Campus, Gold Coast, Queensland QLD 4222, Australia. ${ }^{2}$ School of Medical Science, Griffith University, Gold Coast Campus, Gold Coast, Queensland QLD 4222, Australia. ${ }^{3}$ Department of Microbiology, University of Tennessee, Knoxville, Tennessee 37996, USA. ${ }^{4}$ Computer Science and Mathematics Division, Oak Ridge National Laboratory, Oak Ridge, Tennessee 37861, USA. * These authors contributed equally to this work. Correspondence and requests for materials should be addressed to V.K. (email: v.korolik@Griffith.edu.au).
} 
C ampylobacter jejuni is now recognized as the leading cause of bacterial gastroenteritis in humans throughout the world, representing a considerable drain on economic and public resources ${ }^{1,2}$. The symptoms of campylobacteriosis range from asymptomatic to severe enteritis characterized by fever, severe abdominal cramping and diarrhea with blood and mucus ${ }^{3}$. The bacterium naturally colonizes the gastrointestinal tract of birds and animals, resulting in a commensal relationship, however, consumption of undercooked poultry meat or other food products crosscontaminated during food preparation, give rise to human infections $^{3,4}$. Although the molecular details of $C$. jejuni mediated human pathogenesis are not well understood, results of intestinal biopsies of patients and infected primates together with experimental infection of cultured human intestinal epithelial cells, have demonstrated that C. jejuni damages and invades host epithelial cells ${ }^{5-8}$.

In the past, research has been focused on the understanding of virulence factors involved in bacterial adhesion, invasion, and cytotoxin production. Some of the known putative virulence factors include fibronectin-binding protein $(\mathrm{CadF})^{9}$, cytolethal distending toxin ${ }^{10}$, campylobacter invasion antigens (Cia proteins) ${ }^{11}$, chemotaxis mediated motility ${ }^{12}$, lipoprotein $(\mathrm{JlpA})^{13}$, a phase-variable capsule ${ }^{14}$ and the pVir plasmid ${ }^{15}$. While C. jejuni is known to colonize the intestinal mucus, particularly within the cecal crypts, and is able to overcome the physical and immunological barrier posed by the intestinal mucus layer in order to establish an infection in humans ${ }^{16}$, not much is known on how C. jejuni targets the mucus lining of the small and large intestine of both birds and mammals. The motility and corkscrew morphology of C. jejuni is thought to allow it to penetrate the mucus layer ${ }^{17}$ and mucin and $\mathrm{L}$-fucose are known chemoattractants for C. jejuni ${ }^{12}$. More recently, the interactions of $C$. jejuni with mucus and the mucin glycoproteins, the main component of mucus, have been investigated. C. jejuni was always considered an asaccharolytic organism, however, studies have revealed that certain strains of $C$. jejuni can uptake and metabolize the sugar L-fucose, one of the sugars present on mucin glycoproteins ${ }^{18,19}$.

Motility and chemotaxis have been shown to be critical for $C$. jejuni infection and colonization of its hosts ${ }^{20}$. Chemotaxis receptors, also referred to as chemoreceptors, are important in the activation of the chemotaxis signal transduction cascade. Chemotaxis receptors are called methyl-accepting chemotaxis proteins, or transducer-like proteins, and detect various stimuli in the environment and transduce signals into the cytoplasm modulating the rotational directionality of the flagellar motor ${ }^{21}$. Although tens of thousands of chemoreceptor sequences are available in current databases, ligand-binding specificity is known for only a small number of these proteins. The majority of C. jejuni laboratory strains, such as C. jejuni subsp. jejuni strains NCTC 11168 (ATCC 700819) and ATCC 81116, have 10 chemoreceptors (data from the MiST database ${ }^{22}$ ). Six of these chemoreceptors belong to the class I membrane topology group $^{23}$, where two transmembrane regions demarcate a periplasmic ligand-binding domain. Recently, we and others have identified directly binding ligands for three such $C$. jejuni chemoreceptors: CcaA (Tlp1), CcmL (Tlp3) and Tlp7 (refs 24-26). The receptor specificities ranged from a single ligand (aspartate for $\mathrm{CcaA}$ ) to multiple different and distinct ligands $(10 \text { in total for } \mathrm{CcmL})^{24-26}$. Previous screening of a range of human, avian and newly obtained clinical isolates showed the presence of different subsets of tlp genes coding for class I (group A) chemoreceptors in different strains ${ }^{27}$. Tlp11 is a recently identified chemoreceptor present in strains that were known to lead to disease requiring hospitalization ${ }^{27}$.
In this study, we characterize the Tlp11 chemosensory receptor (which we propose to rename CcrG), identifying the receptor specific ligand, galactose, and showing its recent evolutionary origins and occurrence in only a few, virulent strains. We also study the role for this receptor in adherence to host cells and colonization of chickens.

\section{Results}

Ligand-binding specificity of Tlp11. The Tlp11 sensory receptor was found in approximately $11 \%(5 / 44)$ of $C$. jejuni isolates from humans and chickens via PCR analysis using tlp11 gene specific primers (Supplementary Table 1). The five strains carrying tlp11 were invasive or highly invasive. However, tlp11 could not be found in six C. jejuni strains previously described as hyperinvasive $\mathrm{e}^{28}$.

To determine ligand specificity of Tlp11, the periplasmic sensory domain of this gene, Tlp $11^{\text {peri }}$, was cloned distal to a Histag in an expression vector pET-19b (plasmids described in Supplementary Table 2). The recombinant protein was expressed and purified. Ligand-binding specificity of the His-tagged Tlp11 $1^{\text {peri }}$ was assessed using amino acid, glycan and small molecule arrays. No binding was observed on the chemotaxis ligand array (for the full list of amino acids and salts see Methods), but binding was noted to terminal galactose structures (Table 1, for the full range of glycans tested see Supplementary Table 3). The binding was observed to any free terminal galactose regardless of underlying linkage, however, galactose with a further distal sugar residue bound, such as a sialic acid or fucose, was not recognized (Table 1 ). Furthermore, no terminal $\mathrm{N}$-acetylgalactosamine structures were recognized. Surface plasmon resonance of free galactose, glucose and ribose was performed. Glucose and ribose were included to confirm the absence of binding to these

Table 1 | Glycan structures recognized by CcrG.

\begin{tabular}{|c|c|}
\hline Glycan & Structure \\
\hline $1 \mathrm{~A}$ & Galß1-3GlcNAc \\
\hline $1 \mathrm{~B}$ & Galß1-4GlcNAc \\
\hline $1 C$ & Galß1-4Gal \\
\hline 1D & Galß1-6GlcNAc \\
\hline $1 \mathrm{E}$ & Gal $\beta 1-3 G a l N A c$ \\
\hline $1 \mathrm{~F}$ & Gal $\beta 1-3 G$ alNAc $\beta 1-4 G a l \beta 1-4 G \mid c$ \\
\hline $1 \mathrm{G}$ & Gal $\beta 1-3 G|c N A c \beta 1-3 G a l \beta 1-4 G| c$ \\
\hline $1 \mathrm{H}$ & Gal $\beta 1-4 \mathrm{Glc} N A c \beta 1-3 \mathrm{Gal} \beta 1-4 \mathrm{Glc}$ \\
\hline 11 & Gal $\beta 1-4$ GIcNAc $\beta 1-6($ Gal $\beta 1-4 G \mid c N A c \beta 1-3)$ Gal $\beta 1-4 G \mid c$ \\
\hline $1 \mathrm{~J}$ & Gal $\beta 1-4$ GIcNAc $\beta 1-6$ (Gal $\beta 1-3 G I c N A c \beta 1-3)$ Gal $\beta 1-4 G \mid c$ \\
\hline $1 \mathrm{~K}$ & Gal $\alpha 1-4$ Gal $\beta 1-4 \mathrm{Glc}$ \\
\hline $1 \mathrm{M}$ & Galß1-3GalNAc $\alpha 1-O-S e r$ \\
\hline $1 \mathrm{~N}$ & Gal $\alpha 1-3 \mathrm{Gal}$ \\
\hline 10 & Gal $\alpha 1-3$ Gal $\beta 1-4 G \mid c N A c$ \\
\hline $1 \mathrm{P}$ & Gal $\alpha 1-3 \mathrm{Gal} \beta 1-4 \mathrm{Glc}$ \\
\hline $2 \mathrm{~A}$ & Gal $\alpha 1-3 \mathrm{Gal} \beta 1-4 \mathrm{Gal} \alpha 1-3 \mathrm{Gal}$ \\
\hline $2 B$ & Galß1-6Gal \\
\hline $2 \mathrm{E}$ & Gal 1 1-4Galß1-4GIcNAc \\
\hline $2 \mathrm{G}$ & Gal $\beta 1-3 G I c N A c \beta 1-3$ Gal $\beta 1-4 G \mid c N A c \beta 1-6$ \\
\hline & (Gal $\beta 1-3 G \mid c N A c \beta 1-3)$ Gal $\beta 1-4 G l c$ \\
\hline $2 \mathrm{H}$ & Gal $\beta 1-3 G|c N A c \beta 1-3 G a l \beta 1-4 G| c N A c \beta 1-3 G a l \beta 1-4 G \mid c$ \\
\hline $7 \mathrm{~B}$ & Gal $\beta 1-3$ (Fuc $\alpha 1-4$ )GlcNAc $\beta 1-3$ Gal $\beta 1-4$ Glc \\
\hline $7 C$ & Gal $\beta 1-4($ Fuc $\alpha 1-3)$ GlcNAc $\beta 1-3$ Gal $\beta 1-4 \mathrm{Glc}$ \\
\hline 7D & Fuc $\alpha 1-2$ Gal $\beta 1-3($ Fuc $\alpha 1-4)$ GlcNAc $\beta 1-3 G a l \beta 1-4 G \mid c$ \\
\hline $7 \mathrm{E}$ & Gal $\beta 1-3$ (Fuc $\alpha 1-4$ )GlcNAc $\beta 1-3$ Gal $\beta 1-4($ Fuc $\alpha 1-3)$ Glc \\
\hline $7 \mathrm{H}$ & Gal $\beta 1-4($ Fuc $\alpha 1-3) \mathrm{Glc}$ \\
\hline 71 & Gal $\beta 1-4$ (Fuc $\alpha 1-3$ )GlcNAc \\
\hline 7J & Gal $\beta 1-3($ Fuc $\alpha 1-4)$ GlcNAc \\
\hline $7 \mathrm{M}$ & Gal $\beta 1-3(F u c \alpha 1-2) G a l$ \\
\hline $8 \mathrm{C}$ & Gal $\beta 1-3 G \mid c N A c \beta 1-3 G a l \beta 1-4$ (Fuc $\alpha 1-3$ )GlcNAc $\beta 1-3 G a l \beta 1-4 G \mid c$ \\
\hline
\end{tabular}


glycans since in Escherichia coli the galactose receptor also recognizes glucose and ribose. The disassociation equilibrium constant $\left(K_{\mathrm{D}}\right)$ of Tlp11 ${ }^{\text {peri }}$ for galactose was determined to be $17 \mu \mathrm{M}( \pm 5.7 \mu \mathrm{M})$. No binding to glucose or ribose was detected (Supplementary Fig. 1). Saturation transfer difference (STD) NMR analyses were also undertaken as previously described ${ }^{24}$ to ensure the specificity of the Tlp11-galactose binding. Tlp $11^{\text {peri }}$ binding to galactose, but not glucose or aspartate, which were included as nonbinding controls, was detected (Supplementary Fig. 2). As for the glycan array, surface plasmon resonance and STD-NMR identified galactose as the only ligand for Tlp11. Therefore, hereafter we refer to this receptor as CcrG-Campylobacter ChemoReceptor for Galactose

CcrG origins and phyletic distribution. BLAST analysis of the full length $c c r G$ gene showed 95-98\% identity at the nucleotide level (100\% identity in the periplasmic domain region) among $c c r G$ sequences in all strains that encode this receptor. The search for CcrG orthologs was conducted not only with the full length sequence as a query, but also with the sequences corresponding to the periplasmic ligand-binding domain ( $\left.\mathrm{CcrG}{ }^{\text {peri }}\right)$, and the cytoplasmic signalling domain as separate queries. Full length, $\mathrm{CcrG}^{\text {peri }}$ and the cytoplasmic signalling domain queries all resulted in nearly identical distributions of BLAST hits thus unambiguously identifying CcrG orthologs only in a small subset of strains: three other C. jejuni strains, two strains of Campylobacter coli and one strain of Campylobacter upsaliensis (Supplementary Fig. 3). The closest relative of CcrG is the chemoreceptor annotated as TlpA in C. coli, which is present in many Campylobacter strains, including the widely used laboratory strain 81116, as well as in Helicobacter strains (Supplementary Fig. 3). Thus, CcrG appears to be the TlpA paralog, which arose through recent $t p A$ duplication. Because the function of TlpA is unknown, we have compared CcrG with other class I chemoreceptors in C. jejuni, whose function is known-the aspartate chemoreceptor $\mathrm{CcaA}^{24}$ and the multi-ligand chemoreceptor $\mathrm{CcmL}^{25}$. Interestingly, the $\mathrm{CcrG}$ periplasmic ligand-binding domain (residues 32 to 332 in CcrG) shares 35\% identity with $\mathrm{CcaA}$ and $20 \%$ identity with $\mathrm{CcmL}$, however, the cytoplasmic signalling domain (residues 376 to 707 in CcrG) shares $90 \%$ identity with $\mathrm{CcmL}$ and only $64 \%$ identity with $\mathrm{CcaA}$. The CcrG/TlpA type of chemoreceptors may have originated through a domain swap at some point in the evolution of the Campylobacter/Helicobacter clade. Because of its recent appearance and low sequence identity to known chemoreceptors, we have carried out a detailed sequence analysis of the CcrG ligand-binding region.

Sequence analysis of the CcrG ligand-binding region. Sequence-based searches against the $\mathrm{Pfam}^{29}$ and $\mathrm{CDD}^{30}$ domain databases revealed no known domains in the CcrG periplasmic region (residues 32 to 332); however, similar searches with the corresponding region from its closest homologues (for example, YP_008623181 and YP_008746227) did reveal the dCache_1 domain (Pfam accession number PF02743), suggesting its presence in CcrG. We then used a more sensitive, profileprofile search against the same domain databases using the HHPred tool ${ }^{31}$, which has confidently ( $>99.7 \%$ probability) identified the dCache_1 domain not only in all CcrG orthologs, but also in all homologous chemoreceptors, including $\mathrm{CcmL}$ and CcaA. HHPred searches against protein structures from the PDB database identified the structure of the ligand-binding region of the Vibrio cholerae chemoreceptor (PDB code 3C8C) as the most closely related to $C$. jejuni chemoreceptors. Consequently, we have constructed a multiple sequence alignment of these regions to reveal potential structural similarities and differences between them. While the overall predicted structure of $\mathrm{CcmL}$ follows that of the known 3C8C structure, both CcrG and CcaA have distinct deviations, especially in the $\mathrm{N}$-terminal region corresponding to the membrane-distal subdomain (Fig. 1), which is the site for
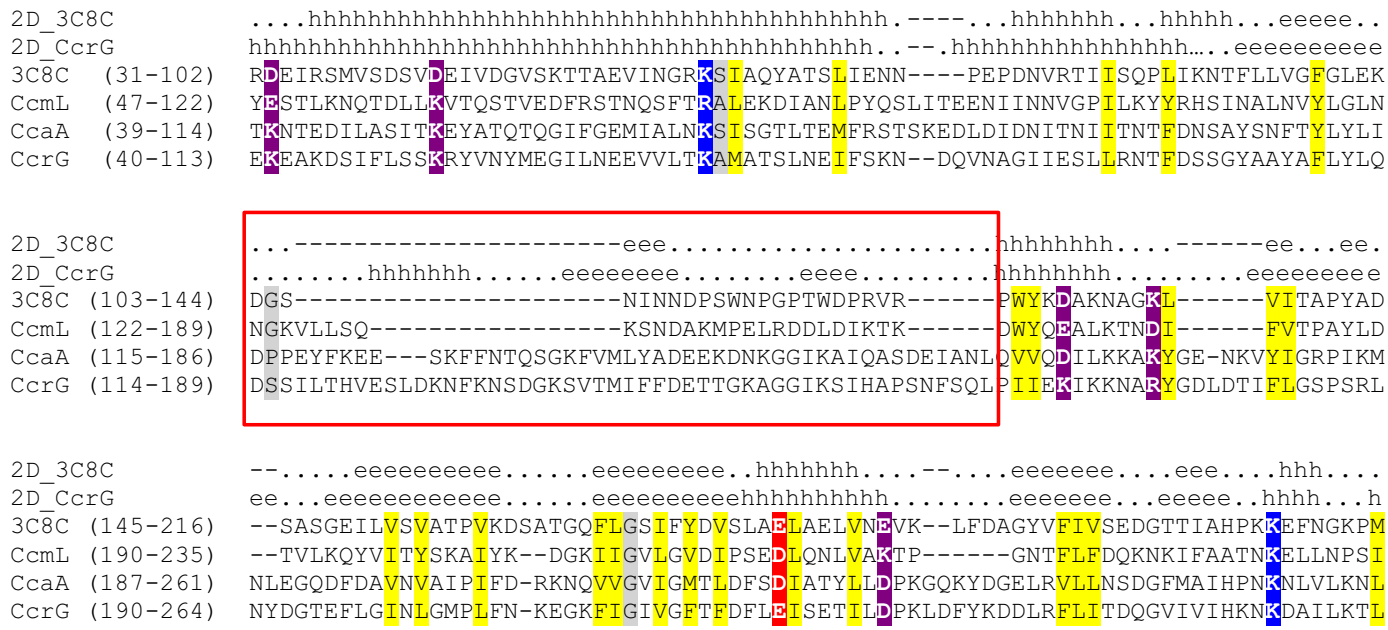

Figure 1 | Periplasmic regions of selected chemoreceptors. Multiple sequence alignment of the periplasmic region from the Vibrio cholerae Mcp37 chemoreceptor (PDB: 3C8C) with those from C. jejuni chemoreceptors. Known (3C8C) and predicted (CcrG) secondary structure elements are shown above the alignment: h, alpha-helix; e, beta strand. Conserved residues are highlighted in yellow (hydrophobic), purple (charged), blue (positively charged), red (negatively charged) and grey (small). Regions of poor conservation are shown within a red box. Locus tag numbers: 3C8C, VCA0923; CcaA, Cj1506c; CcmL, Cj1564; CorG,N135_00253. 
ligand binding in 3C8C. In order to survey all known ligands for dCache_1 domains, we have searched the National Center for Biotechnology Information (NCBI) PubMed records for gene/ protein identifiers for all proteins in the NCBI protein database that contain dCache_1 domains. Table 2 contains information on chemoreceptors and histidine kinases, where ligands were shown to be directly bound by extracellular regions containing the dCache_1 domain. The results show that dCache_1 domains bind amino acids and to a lesser extent organic acids. We have found no reports of a sugar being a main ligand for a dCache_1 domain. We further investigated the ligand-binding regions of dCache_1 containing Group A chemoreceptors through phylogenetic methods. The results of our phylogenetic reconstruction (Supplementary Fig. 3) show that periplasmic regions from dCache_1 Group A chemoreceptors conform to one of two types in C. jejuni, C. coli and C. upsaliensis: CcmL-like receptors (Tlp2, Tlp3 (CcmL) and Tlp4) and CcaA-like receptors (which comprise Tlp1 (CcaA) and Tlp11 (CcrG)). Furthermore, and most striking, the residues in these receptors that correspond to ligand-binding residues identified in 3C8C are differently conserved (either by identity and/or biochemical properties) between the two types (Fig. 1).

Interrogation of 169 C. jejuni genomes showed that, while $C$. jejuni has the ability to synthesize galactose from other metabolites, no galactose catabolic genes could be found in any of the C. jejuni sequences. Additionally, none of the CcrG containing strains had any genes that had homology to proteins for uptake of galactose or other sugars when using the common amino acid domains as the query sequence.

Effects of CcrG on autoagglutination. The presence of $c c r G$ had significant effects on autoagglutination of the harbouring strain. In C. jejuni strain 520, the insertionally inactivated $520 \Delta c c r G:: \mathrm{Km}^{\mathrm{R}}$ showed, on average, a 2.5 -fold reduction in agglutination compared with the wild-type 520 strain, while the allelic insertion $81116 \Omega c c r G$ showed a twofold increase in agglutination compared with the wild-type 81116. The complemented mutant strain $520 \Delta c c r G:: \mathrm{Km}^{\mathrm{R}} \Omega c c r G:: \mathrm{Cat}^{\mathrm{R}}$ (abbreviated to $520 \Delta c c r G \Omega c c r G$ from here onwards) showed full complementation of the mutant phenotype $(P=0.09$; $t$-test $)$ compared with wild-type 520. This indicates that CcrG positively influences C. jejuni autoagglutination (Fig. 2a). This is not the first Class I chemoreceptor to have a role in bacterial agglutination, as CcmL was found to negatively influence autoagglutination of C. jejuni.

Chemotaxis of $C$. jejuni isogenic strains with and without CcrG. Chemotactic responses of the isogenic strains, with and without $\mathrm{CcrG}$, to galactose were determined. Assays were performed using the C. jejuni 520 wild-type strain, insertionally inactivated $520 \Delta c c r G:: \mathrm{Km}^{\mathrm{R}}$ isogenic mutant, the complemented mutant strain $520 \Delta c c r G \Omega c c r G$, the wild-type 81116 strain and its allelic insertion isogenic strain $81116 \Omega c c r G$, as well an invasive human C. jejuni strain FF34 and its allelic insertion isogenic strain

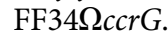

The C. jejuni $520 \Delta c c r G:: \mathrm{Km}^{\mathrm{R}}$ isogenic mutant had significantly reduced chemotactic motility towards galactose (by three orders of magnitude; $P<0.05 ; t$-test), as compared with the wild-type $C$. jejuni 520 and the complemented mutant strain $520 \Delta c c r G \Omega c c r G$ (Fig. 2b). To ensure that the change in chemotaxis towards galactose was not due to a motility defect, we measured chemotaxis towards positive chemotactic motility controls, mucin and aspartate; we found that chemotaxis towards these positive controls was not affected by deletion of CcrG. A FlaAB non-motile mutant was used as a negative control. It is interesting to note that when the $c c r G$ allele was added to the genomes of C. jejuni 81116 and FF34 that normally lack this receptor, the chemotaxis towards galactose was increased by two orders of magnitude (Fig. 2b, $P<0.05$; $t$-test) demonstrating that movement towards galactose was CcrG dependent.

In vitro adherence and invasion assays. To assess the biological significance of CcrG in C. jejuni strains, we compared their adherence to and invasion of polarized Caco-2 cells and the human colorectal cancer cell line HCT116. We tested the following strains: $520 \Delta c c r G:: \mathrm{Km}^{\mathrm{R}}$ (isogenic inactivation) $520 \Delta c c r G \Omega c c r G$ (complemented isogenic inactivation), $81116 \Omega c c r G$ and FF34 $\Omega c c r G$ (allelic addition) mutants, in comparison with wild-type strains 520, 81116 and FF34. Adherence and invasion into HCT116 cells with and without

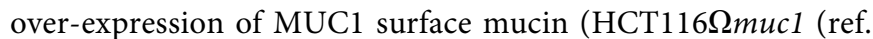
32), rich in galactose, fucose and sialic acid glycosylation was also assessed. Adherence and invasion assays were also performed in competition with free galactose. Expression of CcrG in 520 wild-type, $520 \Delta c c r G \Omega c c r G, 81116 \Omega c c r G$ and FF34 $\Omega c c r G$ strains resulted in higher adherence compared with $C$. jejuni

Table 2 | Ligands directly binding to dCache_1 domain-containing periplasmic regions of bacterial receptor proteins.

\section{Receptor}

McpB, Bacillus subtilis (BSU31260) (ref. 46)

McpC, Bacillus subtilis (BSU13950) (ref. 47)

KinD, Bacillus subtilis (BSU13660) (ref. 48)

PctA, Pseudomonas aeruginosa (PA4309) (ref. 49)

PctB, Pseudomonas aeruginosa (PA4310) (ref. 49)

PctC, Pseudomonas aeruginosa (PA4307) (ref. 49)

Mlp24, Vibrio cholera (VC2161) (ref. 50)

Mlp37, Vibrio cholerae (VCA0923) PDB:3C8C

CcaA, Campylobacter jejuni (Cj1506c) (ref. 24)

CcmL, Campylobacter jejuni (Cj1564) (ref. 25)

McpU, Sinorhizobium meliloti (SMc00975) (ref. 51)

\section{Ligands}

Asparagine, aspartate, glutamine, histidine

Proline, threonine, glycine, serine, valine,

alanine, tyrosine, isoleucine, tryptophan, phenylalanine, leucine, histidine

Pyruvate, propionate, butyrate

Arginine, lysine, tyrosine, tryptophan, phenylalanine,

alanine, valine, isoleucine, leucine, methionine, asparagine, serine, cysteine, threonine, histidine, proline, glycine

Arginine, lysine, alanine, methionine, glutamine Histidine, proline, gamma-aminobutyrate (GABA)

Serine, arginine, asparagine, proline

Alanine

Aspartate

Isoleucine, lysine, arginine, aspartate, glucosamine, succinate, malate, fumarate, $\alpha$-ketoglutarate, purine, thiamine, Proline, histidine, lysine

*Locus tag identifiers for each protein are provided. 
a

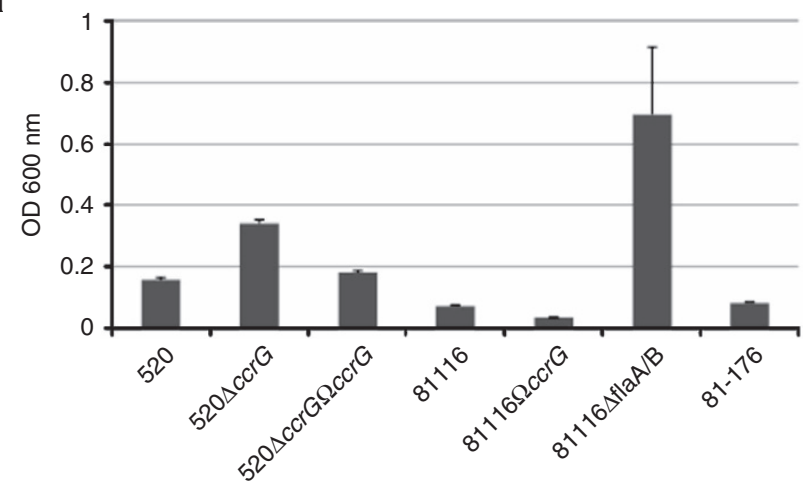

b

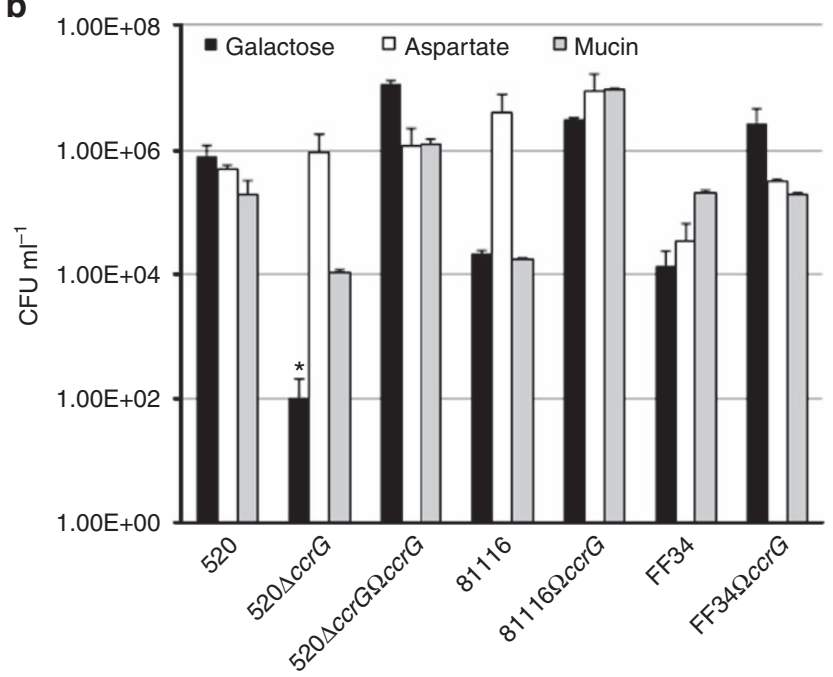

Figure 2 | Effects of CcrG on autoagglutination and chemotaxis.

Autoagglutination and chemotaxis assays of wild-type C. jejuni strains 520 and 81116, mutant ( $520 \Delta c c r G)$, complemented mutant $(520 \Delta c c r G \Omega c c r G)$ and knock-in ( $81116 \Omega \mathrm{ccrG}$ ). (a) Autoagglutination was tested for each of the strains with $C$. jejuni strain 81-176 used as a positive control and C. jejuni non-motile mutants $(81116 \Delta f / a A / f l a B)$ used as a negative control. Data is displayed as the $O D_{600 n m}$ of the supernatant with all strains starting from the same $\mathrm{OD}_{600 \mathrm{~nm}}$. The change observed at the $\mathrm{OD}_{600 \mathrm{~nm}}$ indicates a change in autoagglutination. (b) Chemotaxis assays were performed using aspartate and mucin as positive motility controls with galactose as the test compound. The $C$. jejuni non-motile mutant $(81116 \Delta$ flaA/flaB) was used as the negative control. No movement was observed to galactose and a no-treatment control. Data is from three replicate experiments. The asterisk $\left(^{\star}\right)$ indicates significant difference compared with wild-type strain $(P<0.05 ; t$-test $)$. s.d. errors are shown as bars above the mean.

strains not expressing CcrG in all cell types tested $(P<0.05$; $t$-test; Fig. 3). MUC1 over-expression in HCT116 cells also resulted in higher adherence of all strains expressing CcrG, when compared with CcrG expressing strains adhering to regular HCT116 cells $(P<0.05$; $t$-test Fig. 3$)$, indicating that CcrG plays a role in sensing components of mucin. Addition of $2 \mathrm{mM}$ galactose to the media during adherence assays nullified the increased adherence of CcrG expressing strains, while not significantly affecting the adherence of $C$. jejuni strains not expressing CcrG. CcrG expression was not linked to any significant change in invasion of Caco-2 cells, HCT116 cells or HCT116 cells over-expressing MUC1 (Fig. 3b,d,f).

Colonization potential of $C$. jejuni wild-type and mutant strains. Approximately $48 \mathrm{~h}$ after hatching, chickens were orally infected with different doses of $C$. jejuni 520 wild type, $520 \Delta c c r G:: \mathrm{Km}^{\mathrm{R}}$, 81116 wild type and $81116 \Omega c c r G$ strains. At 5 days post infection, different strains of $C$. jejuni in the caeca were enumerated. The 520 wild type and $520 \Delta c c r G:: \mathrm{Km}^{\mathrm{R}}$ were able to colonize all chickens with the inocula of $1 \times 10^{8}, 1 \times 10^{6}$ and $1 \times 10^{4}$ colonyforming unit (c.f.u). The bacterial loads of the 520 wild type in the caeca ranged from $9.2 \times 10^{7}$ to $7.9 \times 10^{7}$ c.f.u. per gram of caecal content while bacterial loads of the $520 \Delta c c r G:: \mathrm{Km}^{\mathrm{R}}$ ranged from $4.4 \times 10^{6}$ to $7.0 \times 10^{5}$ c.f.u. per gram of caecal content. $520 \Delta c c r G:: \mathrm{Km}^{\mathrm{R}}$ displayed a significant, 10 - to 100 -fold, reduction in colonization when compared with that of the 520 wild type $(P<0.05 ; t$-test $)$. No difference was observed when the colonization potential of chickens by $C$. jejuni 81116 wild type and $81116 \Omega$ ccrG mutant was tested.

The CcrG signalling domain interacts with $\mathrm{CheW}$ and $\mathrm{CheV}$. To further confirm the role of CcrG as a chemoreceptor in C. jejuni 520, a yeast two-hybrid system was used to analyse interactions between the predicted cytoplasmic signalling domain of CcrG and the homologues of the scaffolding proteins of the chemotaxis signalling pathway in C. jejuni, $\mathrm{CheV}$ and $\mathrm{CheW}$, as previously described ${ }^{24}$. Residues $562-707$ of $\mathrm{CcrG}$ ( $\mathrm{CcrG}^{\text {sig }}$ ), encompassing the region homologous to the highly conserved bacterial methyl-accepting chemotaxis protein signalling domain, were interrogated for protein-protein interactions (Supplementary Table 4). This region is almost identical to the predicted cytoplasmic signalling domain of the $C$. jejuni Group A transducer-like proteins of C. jejuni 11168, Tlp2 (Cj0144), CcmL (Cj1564) and Tlp4 (Cj0262). A medium strength interaction was detected between $\mathrm{CcrG}^{\text {sig }}$ and $\mathrm{CheV}$ (AD-CcrG ${ }^{\text {sig }}$ and $\left.\mathrm{BD}-\mathrm{CheV}\right)$. This interaction was also detected in the reciprocal combination of fusion proteins $\left(\mathrm{BD}-\mathrm{CcrG}^{\mathrm{sig}}\right.$ and $\mathrm{AD}-\mathrm{CheV})$. $\mathrm{CcrG}^{\text {sig }}$ was found to interact with the $\mathrm{CheW}$-like domain of $\mathrm{CheV}\left(\mathrm{CheV}^{\mathrm{dW}}\right)$ (AD-CcrG ${ }^{\text {sig }}$ and $\left.\mathrm{BD}-\mathrm{CheV}^{\mathrm{dW}}\right)$. Similarly, $\mathrm{CcrG}^{\text {sig }}$ and $\mathrm{CheW}$ were found to interact in the combination of fusion proteins $\mathrm{BD}-\mathrm{CcrG}^{\text {sig }}$ and $\mathrm{AD}-\mathrm{CheW}$ (Supplementary Table 4). The CcrG signalling domain was also found to interact with itself suggesting dimerization of this chemoreceptor.

\section{Discussion}

In this study, we have shown that CcrG (Tlp11) acts as a directsensing galactose chemoreceptor for C. jejuni 520. Mutation of the $C$. jejuni $c c r G$ gene altered phenotypic characteristics of the bacteria including chemotactic motility and autoagglutination behaviour, suggesting a role for this chemoreceptor in the interaction of $C$. jejuni with its hosts. We demonstrated that the sensory domain of the C. jejuni CcrG chemoreceptor has binding specificity to galactose with the $K_{\mathrm{D}}$ of CcrG to galactose in a biologically relevant range $(17 \mu \mathrm{M})$, similar to those observed for other C. jejuni sensory receptors ${ }^{24,25}$. Increased chemotaxis towards galactose of allelic addition strains, $81116 \Omega c c r G$ and FF $34 \Omega c c r G$, further confirmed that the chemotaxis response to galactose was affected by CcrG. Therefore, we propose to name this receptor CcrG.

In a previous study by Hugdahl et al. ${ }^{12}$, chemotaxis of $C$. jejuni to galactose was not detected. We think it is likely that the strains used in that study did not contain CcrG; so far this receptor has been identified in only a few highly virulent, closely related strains $^{33}$. This fact strongly suggests recent evolutionary origins of CcrG, which is further corroborated by protein sequence analysis.

Bacterial chemoreceptors have unevenly conserved domains. The cytoplasmic signalling domains show extremely high conservation levels due to evolutionary pressure to maintain multiple protein-protein interactions ${ }^{34}$. Conversely, the ligand- 
a

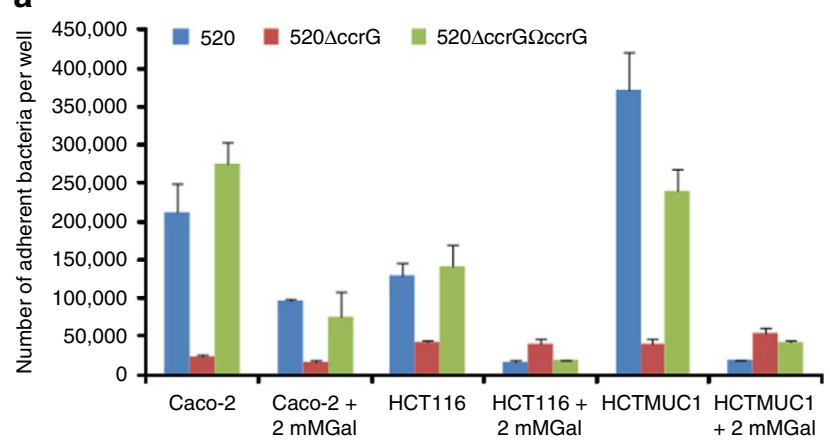

C

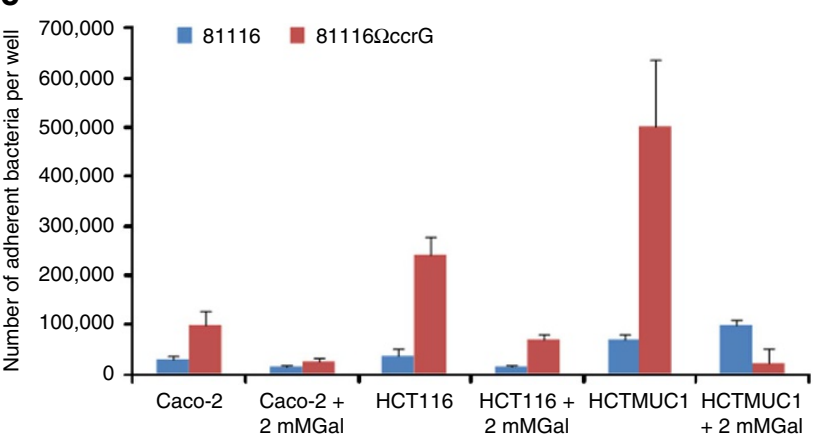

e

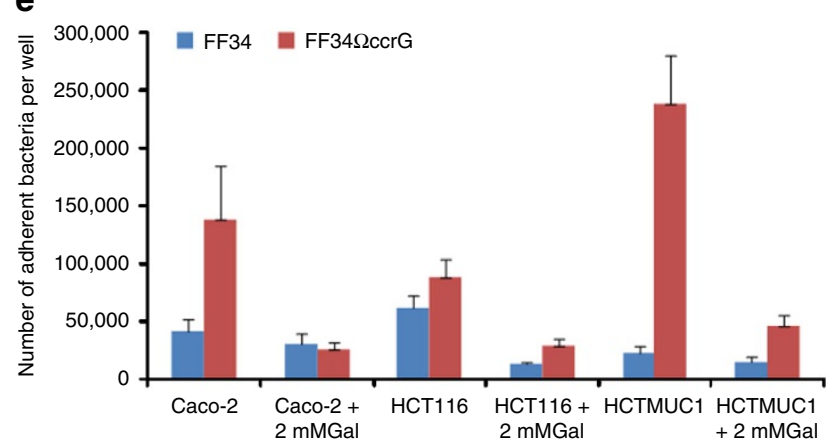

b

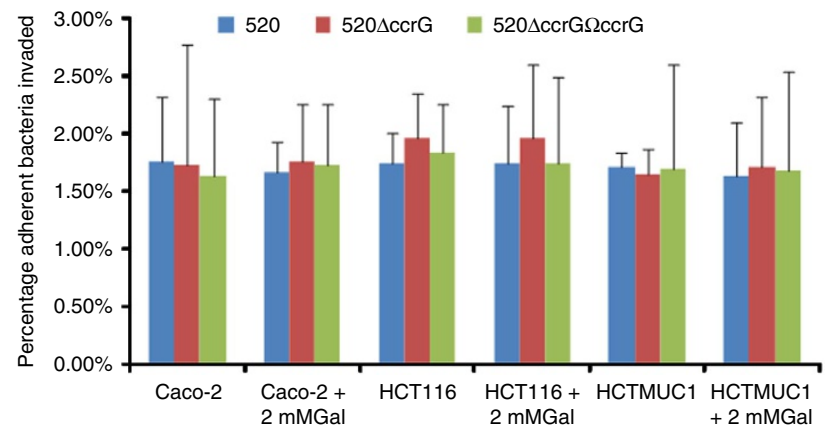

d

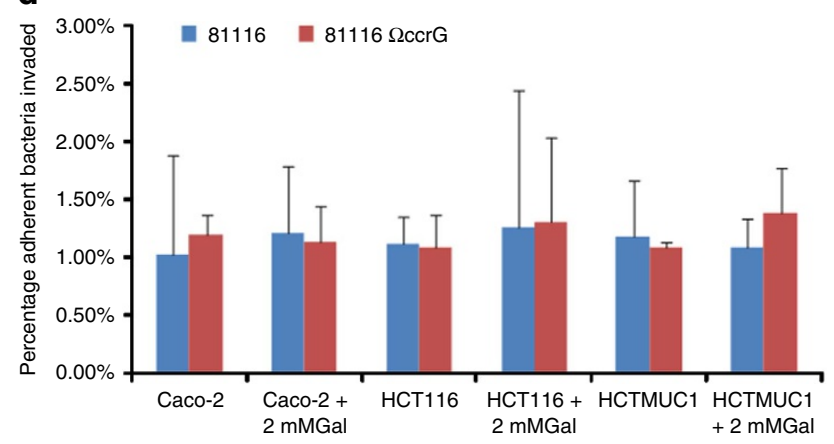

f

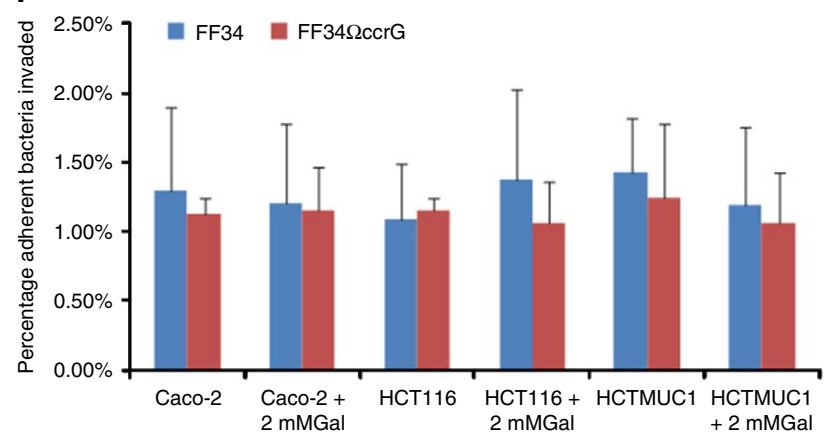

Figure 3 | Adhesion and invasion analysis. Adhesion (a, c, e) and invasion (b, d, f) analysis of Caco2, HCT116 and HCT116 $\Omega$ muc1 cell lines on C. jejuni 520 wild-type, $520 \Delta c c r G:: K m^{R}$, 520 $\Delta c c r G \Omega c c r G$ (a and b), 81116 wild-type, $81116 \Omega c c r G$ (c, d), FF34 wild-type and FF34 $\Omega c c r G$ (e, f) strains. Adhesion and invasion analyses are presented as the mean of invasion from three replicate experiments and standard deviation errors are shown as bars above the mean $(P<0.05$; $t$-test $)$.

binding (sensory) domains detect numerous signals ${ }^{35}$ and are highly variable in sequence, as each chemoreceptor adapts to sense specific physicochemical queues, often after gene duplication $^{35}$. In the case of CcrG, its ligand-binding and signalling domains are most similar to corresponding regions in two different chemoreceptors, suggesting a recent domain swap. Extreme sequence conservation of the CcrG signalling domain and its ability to bind $\mathrm{CheW}$ and $\mathrm{CheV}$ scaffolding proteins suggest that it is an integral part of the chemosensory signalling array identified in C. jejuni ${ }^{36}$.

In C. jejuni, CcrG appears to bind galactose directly and does not bind either glucose or ribose. This is in contrast to the model organism, E. coli, where the galactose/glucose and ribose chemotaxis is mediated by periplasmic sugar-binding proteins interacting with the Trg chemoreceptor ${ }^{37}$. Furthermore, the galactose/ribose binding protein in E. coli belongs to a different structural fold than the dCache_1 domain of the CcrG chemoreceptor further highlighting the novel mechanism for galactose sensing in C. jejuni chemotaxis. dCache_1 domains comprise the largest group of extracellular sensors in bacteria ${ }^{36}$; however, to our knowledge, CcrG is the first dCache_1containing protein to directly bind a sugar as the main ligand. Ligands directly binding to dCache_1 domains in other chemoreceptors and sensory histidine kinases from various bacteria include primarily amino and organic acids (Table 2).

It is interesting to note that $C$. jejuni strain 81116 is chemotactic towards galactose, indicating that there is another receptor or a receptor-periplasmic binding protein pair that may be responsible for sensing galactose in the C. jejuni strains lacking the CcrG receptor. The introduction of the eleventh receptor, CcrG, was able to enhance the chemotactic response of the C. jejuni $81116 \Omega c c r G$ isogenic strain by several orders of magnitude. Moreover, enhanced galactose sensing may contribute, among other factors, to enhanced virulence of $\mathrm{CcrG}$ carrying strains, despite the fact that the presence of CcrG is not correlated with a hyperinvasive phenotype. Additionally, the CcrG encoding $C$. jejuni cells have significantly increased adherence to human cell lines expressing the surface mucin MUC1. The sensing of MUC1 by CcrG could be linked to the increase in adherence as this can be completely blocked by free 
galactose in the media. It is possible that CcrG expressing bacteria sense galactose terminating glycans, which are abundant on MUC1. Consequently, CcrG may have a role in directly sensing host cell surface glycans, thereby potentially enhancing the ability of CcrG expressing strains to cause disease.

Further characterization of the ligands of $C$. jejuni chemosensory proteins will contribute to understanding not only the chemotaxis signalling pathways involved in colonization and invasion, but also the importance of external signals in the survival and pathogenesis of this organism. The identification of a unique Cache domain-containing chemoreceptor, directly sensing galactose, has implications not only for $C$. jejuni chemotaxis but for other bacteria, many of which utilize Cache domains as environmental sensors ${ }^{36}$.

\section{Methods}

Bacterial strains and growth conditions. Strains and plasmids used in this study are described in Supplementary Table 2. C. jejuni 81116 and 11168 were kindly provided by Diane Newell, Veterinary Laboratories Agency, UK. Hyperinvasive strains of $C$. jejuni were kindly provided by Georgina Manning, Nottingham Trent University, UK. C. jejuni and E. coli strains were grown as described previously ${ }^{24}$.

Preparation of CcrG periplasmic sensory domain. Screening of C. jejuni strains for CcrG and PCR for cloning of the DNA sequence encoding the CcrG periplasmic sensory domain, $\mathrm{CcrG}^{\text {peri }}$, into a protein expression vector pET-19b (Novagen) were performed using primers for CcrG-periplasmic region Tlp11mid F: CTC TGA TGG CAA AAG TGT AAC and Tlp11mid R: CTC TTC AGA TTG AGC GAT AAC, previously published in Day et al ${ }^{27}$. The CcrG ${ }^{\text {peri }}$ protein was purified and verified as described for CcaA in Hartley-Tassell et al. ${ }^{24}$.

Identification of protein ligand interactions for CcrG. Glycan and small molecule arrays were performed as described previously ${ }^{24,25}$. Briefly, $1 \mu \mathrm{g}$ of protein precomplexed with anti-His antibody (Cell Signaling) and rabbit anti-mouse/goat anti-rabbit Alexa 555 IgG (Thermo Scientific) in a molar ratio of 1:0.5:0.25 was incubated on the array for $30 \mathrm{~min}$ in phosphate buffered saline (Thermo Scientific). Arrays were scanned and analysed using ScanArray Express (Perkin Elmer). Small molecule array contained: alanine, arginine, asparagine, aspartate, cysteine, fumaric acid, glucosamine, glutamic acid, glutamine, histidine, isoleucine, leucine, lysine, malic acid, methionine, phenylalanine, proline, purine, serine, succinic acid, thiamine, threonine, tryptophan, tyrosine, valine and $\alpha$-ketoglutarate. Biacore and STD-NMR analysis were performed as described in Rahman et al. ${ }^{25}$ with galactose, ribose and glucose used in place of the amino acids in the same concentration range.

Insertional inactivation of ccrG. C. jejuni $520 \mathrm{ccrG}$ coding region was amplified using forward $5^{\prime}$-ATG AAT TTT CGT TCT CTA AAT TTA AG- $3^{\prime}$ and reverse $5^{\prime}$ TTA ATG ATT CTC TTC CTT CTT AAC- $3^{\prime}$ primers. The PCR product was cloned into pGEMT-Easy (Promega). The coding region was interrupted by insertion of a non-polar 1.1-kb kanamycin resistance cassette from a recombinant plasmid pMW10 (ref. 38) into a unique BglII site using BglII flanked primers (forward 5'-AGA TCT GCT CGG AAT TAA CCC TCA C-3' and reverse 5' -AGA TCT CTG TTT TCT GGT ATT TAA G-3'). The isogenic $c c r G$ mutant, $520 \Delta c c r G:: \mathrm{Km}^{\mathrm{R}}$, was constructed by electrotransformation of C. jejuni strain 520 and verified as previously described ${ }^{39}$. The $520 \Delta c c r G:: \mathrm{Km}^{\mathrm{R}}$ mutant was complemented by the insertion of $C$. jejuni $520 \mathrm{ccrG}$ coding region, which was amplified using the forward and reverse primers flanked with BsmBI sites shown above. The PCR product was first cloned into pGEMT-Easy (Promega) and subcloned into pC46 (ref. 40). The $c c r G$ containing pC46 vector was cloned into $520 \Delta c c r G:: \mathrm{Km}^{\mathrm{R}}$ using natural transformation and plated onto plates containing $50 \mu \mathrm{g} \mathrm{ml}^{-1}$ kanamycin and $10 \mu \mathrm{g} \mathrm{ml}^{-1}$ chloramphenicol. The complemented strain $520 \Delta c c r G \Omega c c r G$ was tested by PCR to confirm the presence of the uninterrupted $c c r G$ gene fragment and the chloramphenicol resistance gene. There was no growth rate difference between $C$. jejuni wild-type $520,520 \Delta c c r G:: \mathrm{Km}^{\mathrm{R}}$ or $520 \Delta c c r G \Omega c c r G$ (Supplementary Fig. 4a; $P>0.2$; $t$-test).

Construction of allelic addition isogenic mutants. To create the heterologous gene expression mutant, the entire coding region of the $520 \mathrm{ccrG}$ gene, including the start and stop codons, was ligated directly into pBF6A (pBluscript $\Omega$ C.jejuni::flaA::flaB::Km ${ }^{\mathrm{R}} \mathrm{Amp}{ }^{\mathrm{R}}$; Benjamin Fry, RMIT University, Melbourne, Australia, unpublished) into the non-essential flaB gene in the same orientation and electroporated into $C$. jejuni strains 81116 and FF34, to add a $c c r G$ allele to the genome. A control motility mutant carrying the $\mathrm{Km}^{\mathrm{R}}$ cassette within the C. jejuni strain 81116 flaB gene was similarly constructed and normal motility verified. No difference in growth rate was observed between C. jejuni 81116 wild type and $81116 \Omega c c r G$ strains (Supplementary Fig. $4 \mathrm{~b} ; P=0.85$; $t$-test).

Verification of expression of ccrG alleles. Expression levels of $c c r G$ were tested in $520 \Delta c c r G:: \mathrm{Km}^{\mathrm{R}}$ (isogenic inactivation), FF34 $\Omega c c r G$ and $81116 \Omega c c r G$ (allelic addition) compared with wild-type controls by real-time PCR using primers Tlp11mid F and R described above. C. jejuni $520 \Delta c c r G:: \mathrm{Km}^{\mathrm{R}}$ was found to express $c c r G 1.93$ ( \pm 0.34 ) fold lower than 520 wild-type control. $520 \Delta c c r G \Omega c c r G$ was found to express $c c r G 3.22$ ( \pm 0.42 ) fold above the 520 wild-type control. While the FF34 $\Omega c c r G$ and $81116 \Omega$ ccrG expression levels were 2.47 ( \pm 0.68 ) fold and 2.63 $( \pm 0.29)$ fold higher than 520 wild-type control, respectively. C. jejuni 81116 and FF34 wild-type strains were confirmed to lack $c c r G$ expression.

\section{Adherence and invasion assays of culture cell lines Caco-2 and HCT116.} Caco-2, HCT116 and HCT116 overexpressing MUC1 adherence and invasion assays were performed with and without centrifugation as previously described $^{24,32,41}$. Briefly, cells were grown to confluence in a 24 -well plate and adherence/ invasion assays were performed with a starting inoculum of $\sim 10^{8}$ bacteria per well. Galactose competition was performed by pre-incubating C. jejuni with $2 \mathrm{mM}$ galactose and keeping galactose at $2 \mathrm{mM}$ throughout the adherence assay.

Soft agar motility assay and nutrient depleted chemotaxis assay. Motility and chemotaxis assays were performed as previously described ${ }^{25}$. Briefly, $6 \mathrm{~mm}$ plugs were removed from a $0.5 \%$ agar $\left(\right.$ in $\mathrm{H}_{2} \mathrm{O}$ ) plate and replaced with $0.5 \%$ agar with $2 \mathrm{mM}$ of amino acid, buffer or mucin. The plates were overlaid with $0.1 \%$ agar in $\mathrm{H}_{2} \mathrm{O}$ (no nutritional supplements) and left for $2 \mathrm{~h}$ to allow for diffusion. A $24 \mathrm{~h}$ culture of $C$. jejuni strains, grown at $42^{\circ} \mathrm{C}$, was sub-cultured in Brucella broth for a further $18 \mathrm{~h}$ with shaking at 50 r.p.m. Cells were collected by centrifugation, resuspended in saline and $10^{8}$ c.f.u. of $C$. jejuni in a $100 \mu \mathrm{l}$ drop were then inoculated in the center of the petri dish. Viable bacteria that have migrated to amino acid plugs after $4 \mathrm{~h}$ at $37^{\circ} \mathrm{C}$, were enumerated.

Analysis of the isogenic ccrG mutant in chicken colonization. The 2-day mode for chicken colonization was used ${ }^{42}$ to assay the effects of isogenic $c c r G$ mutation on bacterial colonization potential as previously described ${ }^{24,25}$. Briefly, Groups of 8 to 10 two-day old chicks in isolators were inoculated orally with $10^{6}$ c.f.u. of $C$. jejuni strain 520 or isogenic mutants of this strain. Colonization was monitored daily by culture from cloacal swabs. Chickens were euthanized 5 days following inoculation, and the level of colonization was enumerated.

Yeast two-hybrid analysis. Yeast two-hybrid analysis of protein interactions was performed as described previously ${ }^{24}$. Interactions involving BD-CcrG ${ }^{\text {sig }}$ were analysed on intermediate stringency containing $1 \mathrm{mM} 3-\mathrm{AT}$, as this media was found to suppress the autonomous activation of reporter gene expression exhibited by this fusion protein.

Bioinformatics. BLAST searches ${ }^{43}$ against the RefSeq database at NCBI were carried out with default parameters. Protein domain architectures were obtained from the Pfam $^{29}$ and Conserved Domain ${ }^{30}$ databases. Sensitive profile-profile searches for domain identification were carried out using HHpred ${ }^{31}$. Multiple sequence alignments were constructed using LINS-I algorithm from the MAFFT v7 program $^{44}$. Maximum-likelihood phylogenetic trees were constructed using the MEGA6 package ${ }^{45}$.

Data availability. The data that support the findings of this study are included in this published article and its Supplementary Information files, or are available from the corresponding author upon request.

\section{References}

1. Frost, J. A. Current epidemiological issues in human campylobacteriosis. J. Appl. Microbiol. 90, 85s-95s (2001).

2. Altekruse, S. F., Stern, N. J., Fields, P. I. \& Swerdlow, D. L. Campylobacter jejuni an emerging foodborne pathogen. Emerg. Infect. Dis. 5, 28-35 (1999).

3. Blaser, M. J. Epidemiological and clinical features of Campylobacter jejuni infection. J. Infect. Dis. 176, 103-105 (1997).

4. Shane, S. M. Campylobacter infection of commercial poultry. Rev. Sci. Tech. 19, 376-395 (2000).

5. McCormick, B. A. The use of transepithelial models to examine host-pathogen interactions. Curr. Opin. Microbiol. 6, 77-81 (2003).

6. Russell, R. G., O’Donnoghue, M., Blake, Jr D. C., Zulty, J. \& DeTolla, L. J. Early colonic damage and invasion of Campylobacter jejuni in experimentally challenged infant Macaca mulatta. J. Infect. Dis. 168, 210-215 (1993).

7. Russell, R. G. \& Blake, Jr D. C. Cell association and invasion of Caco-2 cells by Campylobacter jejuni. Infect. Immun. 62, 3773-3779 (1994). 
8. Black, R. E., Levine, M. M., Clements, M. L., Hughes, T. P. \& Blaser, M. J. Experimental Campylobacter jejuni infection in humans. J. Infect. Dis. 157, 472-479 (1988).

9. Konkel, M. E., Garvis, S. G., Tipton, S. L., Anderson, D. E. J. \& Cieplak, W. J. Identification and molecular cloning of a gene encoding a fibronectin-binding protein (CadF) from Campylobacter jejuni. Mol. Microbiol. 24, 953-963 (1997).

10. Johnson, W. M. \& Lior, H. A new heat-labile cytolethal distending toxin (CLDT) produced by Campylobacter spp. Microb. Pathog. 4, 115-126 (1988).

11. Konkel, M. E., Kim, B. J., Rivera-Amill, V. \& Garvis, S. G. Identification of proteins required for the internalization of Campylobacter jejuni into cultured mammalian cells. Adv. Exp. Med. Biol. 473, 215-224 (1999).

12. Hugdahl, M. B., Beery, J. T. \& Doyle, M. P. Chemotactic behavior of Campylobacter jejuni. Infect. Immun. 56, 1560-1566 (1988).

13. Jin, S. et al. JlpA, a novel surface-exposed lipoprotein specific to Campylobacter jejuni, mediates adherence to host epithelial cells. Mol. Microbiol. 39, 1225-1236 (2001)

14. Bacon, D. J. et al. A phase-variable capsule is involved in virulence of Campylobacter jejuni 81-176. Mol. Microbiol. 40, 769-777 (2001).

15. Bacon, D. J. et al. Involvement of a plasmid in virulence of Campylobacter jejuni 81-176. Infect. Immun. 68, 4384-4390 (2000).

16. Ketley, J. M. Pathogenesis of enteric infection by Campylobacter. Microbiology 143(Pt 1), 5-21 (1997).

17. Szymanski, C. M., King, M., Haardt, M. \& Armstrong, G. D. Campylobacter jejuni motility and invasion of Caco-2 cells. Infect. Immun. 63, 4295-4300 (1995).

18. Muraoka, W. T. \& Zhang, Q. Phenotypic and genotypic evidence for L-fucose utilization by Campylobacter jejuni. J. Bacteriol. 193, 1065-1075 (2011).

19. Stahl, M. et al. L-fucose utilization provides Campylobacter jejuni with a competitive advantage. Proc. Natl Acad. Sci. USA 108, 7194-7199 (2011).

20. Yao, R. J., Burr, D. H. \& Guerry, P. CheY-mediated modulation of Campylobacter jejuni virulence. Mol. Microbiol. 23, 1021-1031 (1997).

21. Hazelbauer, G. L., Falke, J. J. \& Parkinson, J. S. Bacterial chemoreceptors: highperformance signaling in networked arrays. Trends Biochem. Sci. 33, 9-19 (2008).

22. Ulrich, L. E. \& Zhulin, I. B. The MiST2 database: a comprehensive genomics resource on microbial signal transduction. Nucleic Acids Res. 38, D401-D407 (2010).

23. Wuichet, K., Alexander, R. P. \& Zhulin, I. B. Comparative genomic and protein sequence analyses of a complex system controlling bacterial chemotaxis. Methods Enzymol. 422, 1-31 (2007).

24. Hartley-Tassell, L. E. et al. Identification and characterization of the aspartate chemosensory receptor of Campylobacter jejuni. Mol. Microbiol. 75, 710-730 (2010).

25. Rahman, H. et al. Characterisation of a multi-ligand binding chemoreceptor CcmL (Tlp3) of Campylobacter jejuni. PLoS Pathogens 10, e1003822 (2014).

26. Tareen, A. M., Dasti, J. I., Zautner, A. E., Gross, U. \& Lugert, R. Campylobacter

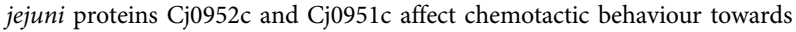
formic acid and are important for invasion of host cells. Microbiology 156, 3123-3135 (2010)

27. Day, C. J. et al. Variation of chemosensory receptor content of Campylobacter jejuni strains and modulation of receptor gene expression under different in vivo and in vitro growth conditions. BMC Microbiol. 12 (2012).

28. Fearnley, C. et al. Identification of hyperinvasive Campylobacter jejuni strains isolated from poultry and human clinical sources. J. Med. Microbiol. 57, 570-580 (2008).

29. Punta, M. et al. The Pfam protein families database. Nucleic Acids Res. 40, D290-D301 (2012).

30. Marchler-Bauer, A. et al. CDD: conserved domains and protein threedimensional structure. Nucleic Acids Res. 41, D348-D352 (2013)

31. Soding, J., Biegert, A. \& Lupas, A. N. The HHpred interactive server for protein homology detection and structure prediction. Nucleic Acids Res. 33, W244-W248 (2005).

32. McAuley, J. L. et al. MUC1 cell surface mucin is a critical element of the mucosal barrier to infection. J. Clin. Invest. 117, 2313-2324 (2007).

33. Korolik, V. \& Ketley, J. M. in Campylobacter (eds Nachamkin, I., Symanski, C. M. \& Blaser, M. J.) 351-366 (ASM Press, 2008).

34. Alexander, R. P. \& Zhulin, I. B. Evolutionary genomics reveals conserved structural determinants of signaling and adaptation in microbial chemoreceptors. Proc. Natl Acad. Sci. USA 104, 2885-2890 (2007).

35. Wuichet, K. \& Zhulin, I. B. Origins and diversification of a complex signal transduction system in prokaryotes. Sci. Signal. 3, ra50 (2010).

36. Upadhyay, A. A., Fleetwood, A. D., Adebali, O., Finn, R. D. \& Zhulin, I. B. Cache domains that are homologous to, but different from PAS domains comprise the largest superfamily of extracellular sensors in prokaryotes. PLoS Comput. Biol. 12, e1004862 (2016).

37. Kondoh, H., Ball, C. B. \& Adler, J. Identification of a methyl-accepting chemotaxis protein for the ribose and galactose chemoreceptors of Escherichia coli. Proc. Natl Acad. Sci. USA 76, 260-264 (1979).
38. Wosten, M. M. S. M., Boeve, M., Koot, M. G. A., van Nuenen, A. C. \& van der Zeijst, B. A. M. Identification of Campylobacter jejuni promoter sequences. J. Bacteriol. 180, 594-599 (1998).

39. Sambrook, J. \& Russell, D. Molecular Cloning: A Laboratory Manual 3rd edn (Cold Spring Harbor Laboratory Press, 2001).

40. Gaskin, D. J. H., Van Vliet, A. H. M. \& Pearson, B. M. The Campylobacter genetic toolbox: development of tractable and generally applicable genetic techniques for Campylobacter jejuni. Zoonoses Public Health 54, 101-101 (2007).

41. Day, C. J. et al. Differential carbohydrate recognition by Campylobacter jejuni strain 11168: influences of temperature and growth conditions. PLoS ONE 4, e4927 (2009).

42. Ringoir, D. D., Szylo, D. \& Korolik, V. Comparison of 2-day-old and 14-dayold chicken colonization models for Campylobacter jejuni. FEMS Immunol. Med. Microbiol. 49, 155-158 (2007).

43. Altschul, S. F. et al. Gapped BLAST and PSI-BLAST: a new generation of protein database search programs. Nucleic Acids Res. 25, 3389-3402 (1997).

44. Katoh, K. \& Standley, D. M. MAFFT multiple sequence alignment software version 7: improvements in performance and usability. Mol. Biol. Evolutn. 30, 772-780 (2013).

45. Tamura, K., Stecher, G., Peterson, D., Filipski, A. \& Kumar, S. MEGA6: Molecular Evolutionary Genetics Analysis version 6.0. Mol. Biol. Evolutn. 30, 2725-2729 (2013).

46. Glekas, G. D. et al. A PAS domain binds asparagine in the chemotaxis receptor McpB in Bacillus subtilis. J. Biol. Chem. 285, 1870-1878 (2010).

47. Glekas, G. D. et al. The Bacillus subtilis chemoreceptor McpC senses multiple ligands using two discrete mechanisms. J. Biol. Chem. 287, 39412-39418 (2012).

48. Wu, R. et al. Insight into the sporulation phosphorelay: crystal structure of the sensor domain of Bacillus subtilis histidine kinase, KinD. Prot. Sci. Prot. Soc. 22, 564-576 (2013).

49. Rico-Jimenez, M. et al. Paralogous chemoreceptors mediate chemotaxis towards protein amino acids and the non-protein amino acid gammaaminobutyrate (GABA). Mol. Microbiol. 88, 1230-1243 (2013).

50. Nishiyama, S. et al. Mlp24 (McpX) of Vibrio cholerae implicated in pathogenicity functions as a chemoreceptor for multiple amino acids. Infect. Immun. 80, 3170-3178 (2012).

51. Webb, B. A., Hildreth, S., Helm, R. F. \& Scharf, B. E. Sinorhizobium meliloti chemoreceptor McpU mediates chemotaxis toward host plant exudates through direct proline sensing. Appl. Environ. Microbiol. 80, 3404-3515 (2014).

\section{Acknowledgements}

This work was partially supported by The Institute for Glycomics and School of Medical Science, Griffith University (V.K.), NH\&MRC grant 1004490 (V.K.) and NIH grant R01GM0722285 (I.Z.).

\section{Author contributions}

V.K. and C.D. conceived and designed this study; experimental work and data analysis were performed by C.D., R.K., L.S., L.H., G.T., T.N., J.W., V.K.; A.F. and I.Z. performed bioinformatics analysis; preparation of the manuscript was performed by C.D. and V.K. with contribution from all other authors.

\section{Additional information}

Supplementary Information accompanies this paper at http://www.nature.com/ naturecommunications

Competing financial interests: The authors declare no competing financial interests.

Reprints and permission information is available online at http://npg.nature.com/ reprintsandpermissions/

How to cite this article: Day, C. J. et al. A direct-sensing galactose chemoreceptor recently evolved in invasive strains of Campylobacter jejuni. Nat. Commun. 7, 13206 doi: 10.1038/ncomms13206 (2016).

This work is licensed under a Creative Commons Attribution 4.0 International License. The images or other third party material in this article are included in the article's Creative Commons license, unless indicated otherwise in the credit line; if the material is not included under the Creative Commons license, users will need to obtain permission from the license holder to reproduce the material. To view a copy of this license, visit http://creativecommons.org/licenses/by/4.0/

(C) The Author(s) 2016 